\title{
Extraction of Newly-built Roads Using Probe Data
}

\author{
Shuto SUGAI ${ }^{\text {a }}$ *, Sho TAKAHASHI ${ }^{\text {a }}$, Mayumi NOGUCHI ${ }^{\text {a }}$, Akira SASAGAWA ${ }^{\text {a }}$ \\ ${ }^{a}$ Geospatial Information Authority of Japan, sugai-s96dc@mlit.go.jp, takahashi-s96b2@mlit.go.jp, noguchi-m96c3@mlit.go.jp, \\ sasagawa-a96dk@mlit.go.jp \\ * Corresponding author
}

Keywords: Probe Data, Map Update

\section{Abstract:}

Geospatial Information Authority of Japan has been providing the Digital Japan Basic Map as fundamental geospatial information of Japan. In order to keep the map data up-to-date efficiently, we considered the method to extract newlybuilt roads from probe data which represent various moving trajectories of smartphone users.

We used the probe data from NAVITIME JAPAN Co., Ltd., obtained from the GPS navigation application for smartphones the company provided. The probe data are recorded while the application is active, and are provided as a data set of points indicating the moving trajectories. The provided data do not include personally identifiable information. The positioning data are basically matched on the road data in the application; but if the application estimates that the users are not on the road, the raw positioning data are recorded. Note that it is difficult to utilize the data for map update directly, because geometric accuracy of the data may not be enough for map update in detail; thus we have focused on extraction of newly-built roads.

The workflow of the extraction is shown in Figure 1. Considering the geometric accuracy of the update sources in the Digital Japan Basic Map, $17.5 \mathrm{~m}$ wide buffers from the road center lines are generated for the following classification. The points within the buffers indicate that the users moved on existing roads in the Digital Japan Basic Map. The points outside the buffers can be the candidates of newly-built roads. After this classification, we examined all the candidate points and evaluated whether each candidate point represented a newly-built road or not.

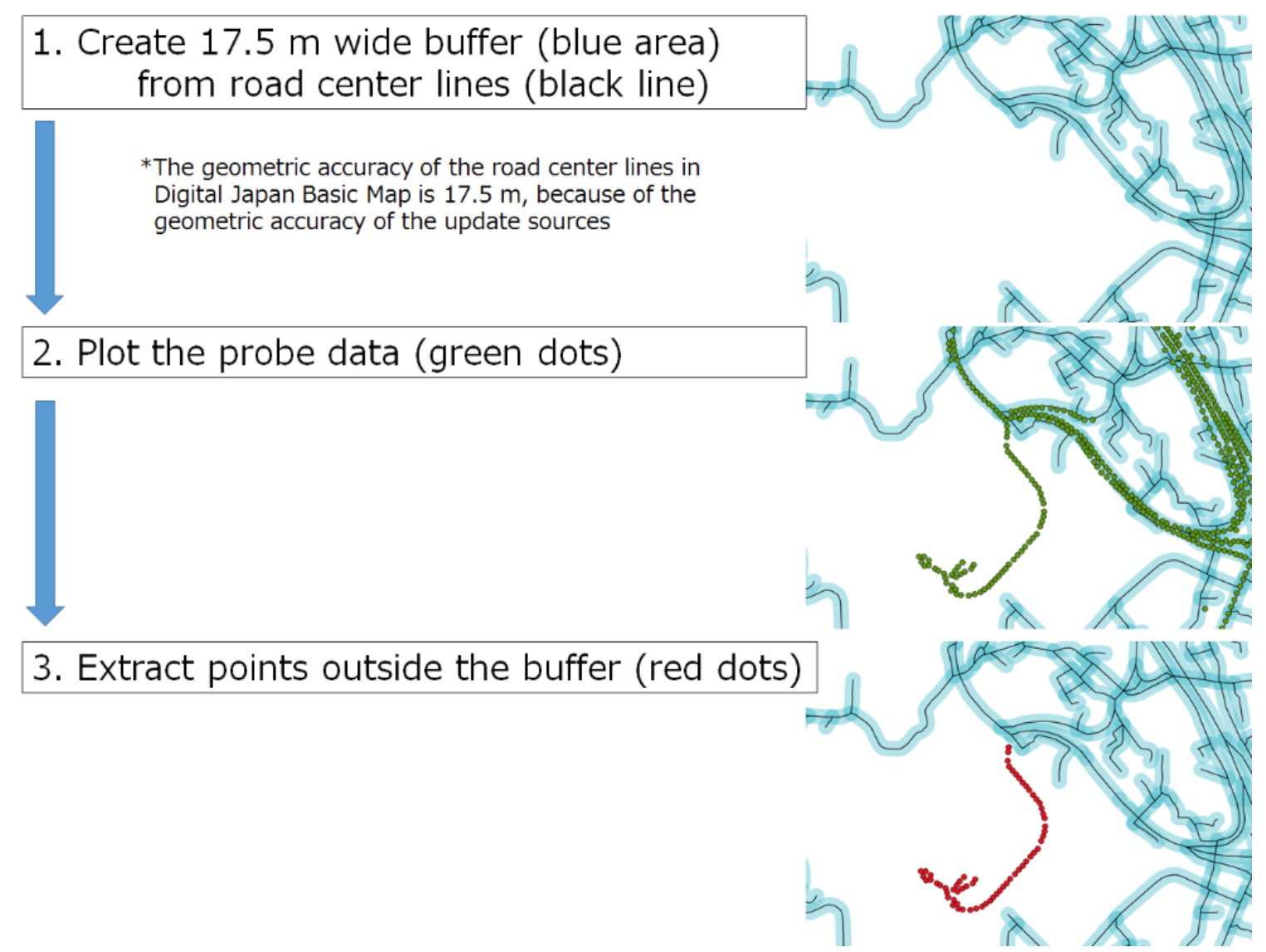

Figure 1. The workflow of extracting newly-built roads from probe data. 
In this study, we used one-week probe data in August 2017 in Fukuoka prefecture, Japan. By the classification described above, we extracted 37,776 candidate points from 871,129 point data. Some examples of the candidate points are shown in Figure 2. In some cases, we succeeded in extracting newly-built roads; in other cases, however, some data were considered to represent movements in buildings or of airplanes. These unnecessary data increase the number of inappropriate candidate points. In order to reduce the number of such inappropriate points, we have tried to introduce a filtering process using the user's velocity. In the conference, the result of this filtering will be reported.

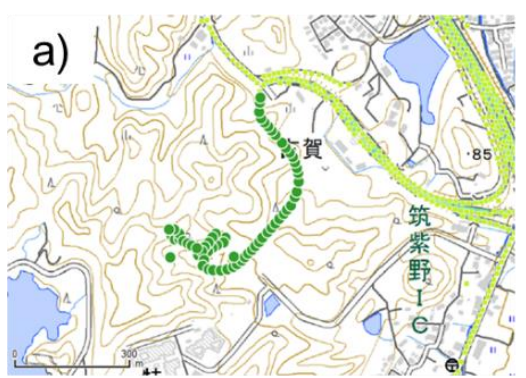

c)

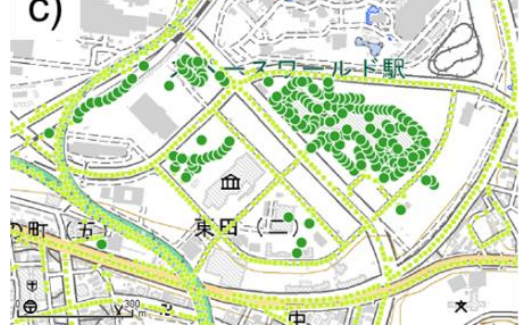

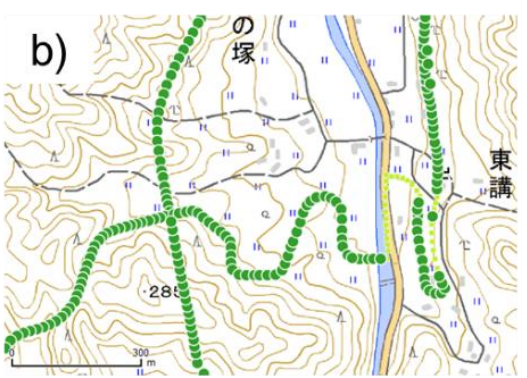

d)

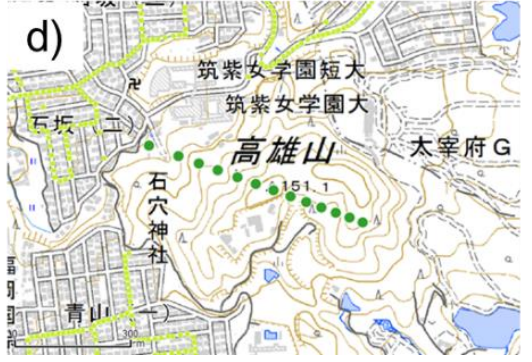

\section{Probe data}

Outside the buffers

Inside the buffers

Figure 2. Examples of extracted probe data: a) and b) indicate successful cases of extracting newly-built roads; c) seems to indicate movements inside a building; d) indicates a movement of an airplane. 